\title{
HAT-P-24b: AN INFLATED HOT JUPITER ON A 3.36 DAY PERIOD TRANSITING A HOT, METAL-POOR STAR*
}

\author{
D. M. Kipping ${ }^{1,2}$, G. Á. Bakos ${ }^{1,10}$, J. Hartman ${ }^{1}$, G. Torres ${ }^{1}$, A. ShPorer ${ }^{3,4}$, D. W. Latham ${ }^{1}$, Géza Kovács ${ }^{5}$, R. W. Noyes ${ }^{1}$, \\ A. W. Howard ${ }^{6}$, D. A. Fischer ${ }^{7}$, J. A. Johnson ${ }^{8}$, G. W. Marcy ${ }^{6}$, B. BéKY ${ }^{1}$, G. Perumpilly ${ }^{1}$, G. A. Esquerdo ${ }^{1}$, \\ D. D. SASSElov ${ }^{1}$, R. P. StefaniK ${ }^{1}$, J. LÁzÁR ${ }^{9}$, I. PAPP ${ }^{9}$, AND P. SÁRI ${ }^{9}$ \\ ${ }^{1}$ Harvard-Smithsonian Center for Astrophysics, Cambridge, MA, USA; dkipping@cfa.harvard.edu \\ ${ }^{2}$ Department of Physics \& Astronomy, University College London, Gower St., London, UK \\ ${ }^{3}$ Las Cumbres Observatory Global Telescope Network, 6740 Cortona Drive, Suite 102, Santa Barbara, CA 93106, USA \\ ${ }^{4}$ Department of Physics, Broida Hall, University of California, Santa Barbara, CA 93106, USA \\ ${ }^{5}$ Konkoly Observatory, Budapest, Hungary \\ ${ }^{6}$ Department of Astronomy, University of California, Berkeley, CA 93106, USA \\ ${ }^{7}$ Department of Astronomy, Yale University, New Haven, CT 06511, USA \\ ${ }^{8}$ Department of Astrophysics, California Institute of Technology, MC 249-17, Pasadena, CA 91125, USA \\ ${ }^{9}$ Hungarian Astronomical Association, Budapest, Hungary \\ Received 2010 August 1; accepted 2010 October 11; published 2010 December 3
}

\begin{abstract}
We report the discovery of HAT-P-24b, a transiting extrasolar planet orbiting the moderately bright $V=$ 11.818 F8 dwarf star GSC 0774-01441, with a period $P=3.3552464 \pm 0.0000071$ days, transit epoch $T_{c}=2455216.97669 \pm 0.00024$ (BJD)11, and transit duration $3.653 \pm 0.025 \mathrm{hr}$. The host star has a mass of $1.191 \pm 0.042 M_{\odot}$, radius of $1.317 \pm 0.068 R_{\odot}$, effective temperature $6373 \pm 80 \mathrm{~K}$, and a low metallicity of $[\mathrm{Fe} / \mathrm{H}]=-0.16 \pm 0.08$. The planetary companion has a mass of $0.681 \pm 0.031 M_{\mathrm{J}}$ and radius of $1.243 \pm 0.072 R_{\mathrm{J}}$ yielding a mean density of $0.439 \pm 0.069 \mathrm{~g} \mathrm{~cm}^{-3}$. By repeating our global fits with different parameter sets, we have performed a critical investigation of the fitting techniques used for previous Hungarian-made Automated Telescope planetary discoveries. We find that the system properties are robust against the choice of priors. The effects of fixed versus fitted limb darkening are also examined. HAT-P-24b probably maintains a small eccentricity of $e=0.052_{-0.017}^{+0.022}$, which is accepted over the circular orbit model with false alarm probability 5.8\%. In the absence of eccentricity pumping, this result suggests that HAT-P24b experiences less tidal dissipation than Jupiter. Due to relatively rapid stellar rotation, we estimate that HAT-P-24b should exhibit one of the largest known Rossiter-McLaughlin effect amplitudes for an exoplanet $\left(\Delta V_{\mathrm{RM}} \simeq 95 \mathrm{~m} \mathrm{~s}^{-1}\right)$ and thus a precise measurement of the sky-projected spin-orbit alignment should be possible.
\end{abstract}

Key words: planetary systems - stars: individual (HAT-P-24) - techniques: photometric - techniques: spectroscopic

Online-only material: machine-readable table

\section{INTRODUCTION}

The understanding of planetary systems has been spearheaded by the study of transiting extrasolar planets (TEPs) in recent years. Eclipses have long offered a key to unlocking the secrets of the heavenly bodies, for example in solar system studies and in the field of eclipsing binaries. For exoplanets, an eclipse offers a door into the inner workings of an alien system hundreds of light years away. As an inherently low probability event, each and every transiting system is precious and timeless to the planetary scientist. Transits continue to offer unprecedented access to an exoplanet's nature and allow for, amongst other things, the determination of the oblateness of a planet (Seager \& Hui 2002; Carter \& Winn 2010), thermal mapping of the planetary surface (Knutson et al. 2007) and accurate planetary radii at the percent level (Charbonneau et al. 2000).

The Hungarian-made Automated Telescope Network (HATNet; Bakos et al. 2004) survey has been one of the principal contributors to the discovery of TEPs. In operation since 2003,

\footnotetext{
* Based in part on observations obtained at the W. M. Keck Observatory, which is operated by the University of California and the California Institute of Technology. Keck time has been granted by NOAO and NASA. ${ }^{10}$ NSF Fellow.

11 Barycentric Julian dates throughout the paper are calculated from Coordinated Universal Time (UTC).
}

it has now covered approximately $14 \%$ of the sky, searching for TEPs around bright stars $(8 \lesssim I \lesssim 14.0)$. HATNet operates six wide-field instruments: four at the Fred Lawrence Whipple Observatory (FLWO) in Arizona and two on the roof of the hangar housing the Smithsonian Astrophysical Observatory's Submillimeter Array, in Hawaii. Since 2006, HATNet has discovered 23 TEPs (with 16 announced or published so far). In this work, we report our 24th discovery, around the relatively bright star previously known as GSC $0774-01441$.

In Section 2, we report the detection of the photometric signal and the follow-up spectroscopic and photometric observations of HAT-P-24. In Section 3, we describe the analysis of the data, beginning with the determination of the stellar parameters, continuing with a discussion of the methods used to rule out non-planetary, false positive scenarios which could mimic the photometric and spectroscopic observations, and finishing with a description of our global modeling of the photometry and radial velocities (RVs). In Section 4, we investigate the variations of our results using different parameter sets with uniform priors to test the robustness of the fitted parameters. We also discuss the effects of fitting- versus fixing-limbdarkening coefficients. In Sections 5-8, we present discussions and analyses of the orbital eccentricity, a possible linear drift in the RVs, the measured mid-transit times and possibilities for future follow-up observations. Finally, we summarize our findings in Section 9. 

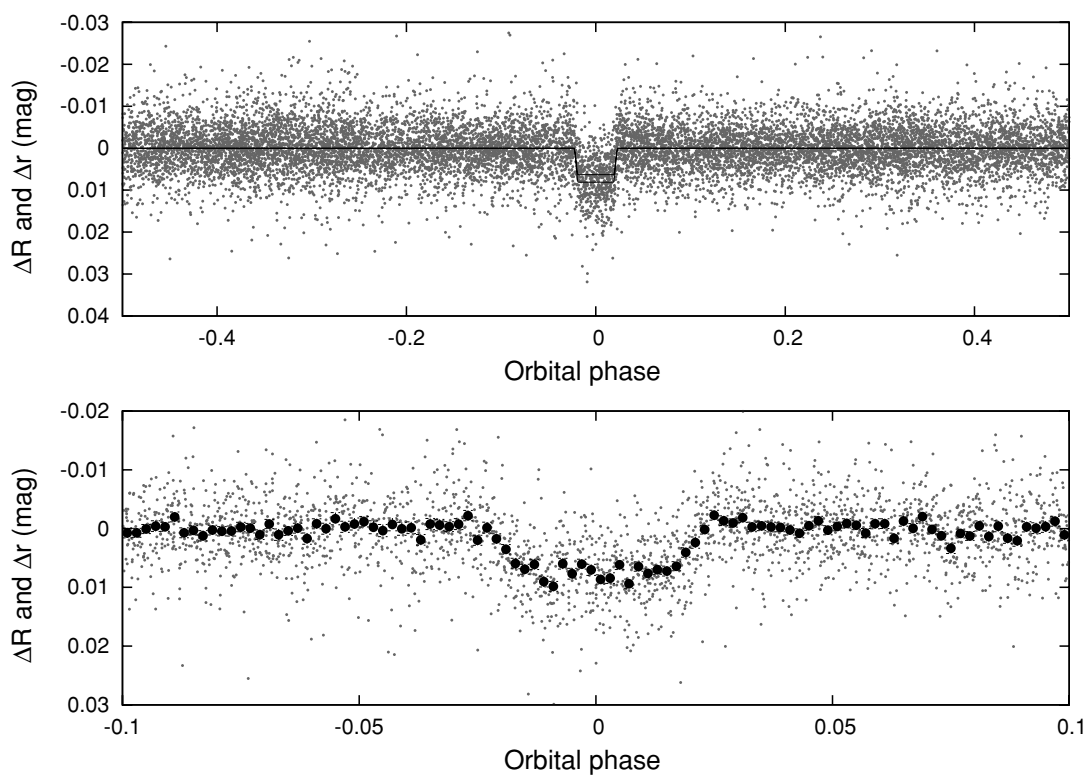

Figure 1. Unbinned light curve of HAT-P-24 including all 14,000 instrumental $R$-band and Sloan $r$-band 5.5 minute cadence measurements obtained with the HAT-5, HAT-6, HAT-8, and HAT-9 telescopes of HATNet (see the text for details), and folded with the period $P=3.3552401$ days resulting from the global fit described in (Section 3). The solid line shows the "P1P3" transit model fit to the light curve (Section 3.3). Solid squares show the 20-point binned light curve.

\section{OBSERVATIONS}

\subsection{Photometric Detection}

The transits of HAT-P-24b were detected with the HAT-5 and HAT- 6 telescopes in Arizona, and with the HAT- 8 and HAT-9 telescopes in Hawaii. The star GSC 0774-01441 lies in the overlap of two fields, internally labeled as $315(07: 30+15: 00)$ and $314(07: 00+15: 00)$. The former field was observed on a nightly basis between 2007 October and 2008 May, while the latter field was observed between 2008 November and 2009 May. For field 315, we gathered $8551 R$-band exposures of 5 minutes at a 5.5 minute cadence, while for field 314 we gathered 5503 Sloan $r$-band images with the same exposure time and cadence. Each field 315 image contained approximately 51,000 stars down to $R \sim 14$, while each 314 image contained approximately 130,000 stars down to $r \sim 14.5$. For the brightest stars in field 315 , we achieved a per-image photometric precision of $3 \mathrm{mmag}$, while for field 314 we achieved $5 \mathrm{mmag}$ precision.

The calibration of the HATNet frames was carried out using standard photometric procedures. For field 314 the calibrated images were then subjected to star detection and astrometry, as described in Pál \& Bakos (2006). Aperture photometry was performed on each image at the stellar centroids derived from the Two Micron All Sky Survey (2MASS; Skrutskie et al. 2006) catalog and the individual astrometric solutions. For field 315, we performed image subtraction photometry following the methods described in Pál (2009b). Aperture photometry was performed on the difference images at the stellar centroid derived from 2MASS, while for the reference flux we adopted the $r$ magnitude of each star, transformed from its 2MASS $J, H$, and $K_{S}$ magnitudes, and made use of the average relation between $r$ and flux measured on the reference image via aperture photometry. The resulting light curves for both fields were decorrelated (cleaned of trends) using the external parameter decorrelation (EPD; see Bakos et al. 2010) technique in "constant" mode and the trend filtering algorithm (TFA; see Kovács et al. 2005). The light curves from each field were independently searched for periodic box-shaped signals using the box least-squares (BLS; see Kovács et al. 2002) method. We detected a significant signal in both of the HATNet light curves of GSC 0774-01441 (also known as 2MASS $07151801+1415453 ; \alpha=07^{\mathrm{h}} 15^{\mathrm{m}} 18^{\mathrm{s}} .00, \delta=+14^{\circ} 15^{\prime} 45^{\prime \prime} .4$; J2000; $V=11.818$ Droege et al. 2006), with an apparent depth of $\sim 7.2 \mathrm{mmag}$ and a period of $P=3.3552$ days (see Figure 1).

\subsection{Reconnaissance Spectroscopy}

As is routine in the HATNet project, all candidates are observed spectroscopically initially to establish whether the transit-like feature in the light curve is of non-planetary origin such a grazing eclipsing binary (i.e., a false positive). For example, large RV variations of the star (tens of $\mathrm{km} \mathrm{s}^{-1}$ ) would indicate such a circumstance.

To perform this task, we used the Harvard-Smithsonian Center for Astrophysics (CfA) Digital Speedometer (DS; Latham 1992); an echelle spectrograph mounted on the FLWO $1.5 \mathrm{~m}$ telescope. This instrument delivers high-resolution spectra $(\lambda / \Delta \lambda \approx 35,000)$ over a single order centered on the $\mathrm{Mg}_{\mathrm{I}} \mathrm{b}$ triplet $(\sim 5187 \AA)$, with typically low signal-to-noise ratios $(\mathrm{S} / \mathrm{N} \sim 10)$ that are nevertheless sufficient to derive RVs with moderate precisions of $0.5-1.0 \mathrm{~km} \mathrm{~s}^{-1}$ for slowly rotating stars. The same spectra can be used to estimate the effective temperature, surface gravity, and projected rotational velocity of the host star, as described by Torres et al. (2002). With this facility we are able to reject many types of false positives, such as $\mathrm{F}$ dwarfs orbited by $\mathrm{M}$ dwarfs, grazing eclipsing binaries, or triple or quadruple star systems.

For HAT-P-24, we obtained five observations with the DS between 2008 May and 2009 January. The velocity measurements showed an rms residual of $0.74 \mathrm{~km} \mathrm{~s}^{-1}$, consistent with no detectable RV variation within the precision of the measurements. All spectra were single-lined, i.e., there is no evidence for additional stars in the system. The atmospheric parameters we infer from these observations are the following: effective temperature $T_{\text {eff } \star}=7000 \pm 100 \mathrm{~K}$, surface gravity $\log g_{\star}=4.5 \pm 0.25$ ( $\left.\log \mathrm{cgs}\right)$, and projected rotational velocity 
Table 1

Relative Radial Velocities, Bisector Spans, and Activity Index Measurements of HAT-P-24

\begin{tabular}{|c|c|c|c|c|c|c|}
\hline $\begin{array}{c}\text { BJD } \\
(2,454,000+)\end{array}$ & $\begin{array}{c}\mathrm{RV}^{\mathrm{a}} \\
\left(\mathrm{m} \mathrm{s}^{-1}\right)\end{array}$ & $\begin{array}{c}\sigma_{\mathrm{RV}}^{\mathrm{b}} \\
\left(\mathrm{ms}^{-1}\right)\end{array}$ & $\begin{array}{c}\mathrm{BS} \\
\left(\mathrm{m} \mathrm{s}^{-1}\right)\end{array}$ & $\begin{array}{c}\sigma_{\mathrm{BS}} \\
\left(\mathrm{m} \mathrm{s}^{-1}\right)\end{array}$ & $S^{\mathrm{c}}$ & $\sigma_{S}^{\mathrm{c}}$ \\
\hline 928.76217 & -49.49 & 6.43 & 1.10 & 1.03 & 0.44 & 0.003 \\
\hline 954.79832 & $\ldots$ & $\ldots$ & -4.52 & 1.35 & 0.43 & 0.003 \\
\hline 955.80587 & -85.15 & 7.37 & -47.96 & 4.39 & 0.42 & 0.008 \\
\hline 956.81494 & -7.84 & 6.61 & -65.72 & 5.91 & 0.45 & 0.005 \\
\hline 963.78272 & 50.62 & 6.41 & 2.42 & 0.68 & 0.45 & 0.003 \\
\hline 1107.14583 & -80.20 & 8.64 & 25.58 & 1.21 & 0.45 & 0.003 \\
\hline 1109.06567 & 48.40 & 7.00 & 8.95 & 0.13 & 0.44 & 0.003 \\
\hline 1112.13808 & 84.68 & 9.02 & 12.27 & 0.08 & 0.43 & 0.003 \\
\hline 1134.10472 & -78.19 & 6.49 & 1.68 & 0.92 & 0.45 & 0.003 \\
\hline 1135.08419 & 55.31 & 5.83 & 8.32 & 0.41 & 0.44 & 0.002 \\
\hline 1136.10198 & 65.26 & 7.53 & 1.35 & 0.77 & 0.43 & 0.002 \\
\hline 1172.94629 & 51.15 & 6.80 & 16.94 & 0.21 & 0.43 & 0.002 \\
\hline 1174.10042 & -85.04 & 6.32 & 7.30 & 0.48 & 0.43 & 0.001 \\
\hline 1187.96722 & -79.11 & 7.17 & 7.57 & 0.35 & 0.40 & 0.002 \\
\hline 1189.07232 & 71.01 & 5.53 & 1.20 & 0.80 & 0.43 & 0.001 \\
\hline 1191.03182 & -80.15 & 5.68 & 5.56 & 0.56 & 0.44 & 0.002 \\
\hline 1192.06936 & $\ldots$ & $\ldots$ & 2.08 & 0.77 & 0.44 & 0.001 \\
\hline 1192.07902 & 37.54 & 5.33 & 3.51 & 0.66 & 0.44 & 0.001 \\
\hline 1193.04199 & 49.79 & 6.10 & 5.20 & 0.59 & 0.43 & 0.002 \\
\hline 1193.85228 & -60.65 & 5.88 & 7.09 & 0.52 & 0.43 & 0.002 \\
\hline
\end{tabular}

Notes. Note that for the iodine-free template exposures we do not measure the RV but do measure the BS and $S$ index. Such template exposures can be distinguished by the missing RV value.

a The zero point of these velocities is arbitrary. An overall offset $\gamma_{\text {rel }}$ fitted to these velocities in Section 3.3 has not been subtracted.

b Internal errors excluding the component of astrophysical jitter considered in Section 3.3.

c Relative chromospheric activity index, not calibrated to the scale of Vaughan et al. (1978).

$v \sin i=11.2 \pm 1.0 \mathrm{~km} \mathrm{~s}^{-1}$. The effective temperature corresponds to an F8 dwarf. The mean heliocentric RV of HAT-P-24 is $\gamma_{\mathrm{RV}}=-2.09 \pm 0.33 \mathrm{~km} \mathrm{~s}^{-1}$. We stress that the DS stellar parameters are based on solar composition models.

\subsection{High-Resolution, High-S/N Spectroscopy}

Given the significant transit detection by HATNet, and the encouraging DS results that rule out obvious false positives, we proceeded with the follow-up of this candidate by obtaining high-resolution, high-S/N spectra to characterize the RV variations, and to refine the determination of the stellar parameters. For this, we used the HIRES instrument (Vogt et al. 1994) on the Keck I telescope located on Mauna Kea, Hawaii, between 2009 April and 2009 December. The width of the spectrometer slit was 0. . 86 , resulting in a resolving power of $\lambda / \Delta \lambda \approx 55,000$, with a wavelength coverage of $\sim 3800-8000 \AA$.

We obtained 18 exposures through an iodine gas absorption cell, which was used to superimpose a dense forest of $I_{2}$ lines on the stellar spectrum and establish an accurate wavelength fiducial (see Marcy \& Butler 1992). An additional two exposures were taken without the iodine cell, for use as templates in the reductions. In practice, we used only the second higher $\mathrm{S} / \mathrm{N}$ template spectrum. Relative RVs in the solar system barycentric frame were derived as described by Butler et al. (1996), incorporating full modeling of the spatial and temporal variations of the instrumental profile. The RV measurements and their uncertainties are listed in Table 1 . The period-folded data, along with a best fit described below in Section 3, are displayed in Figure 2.

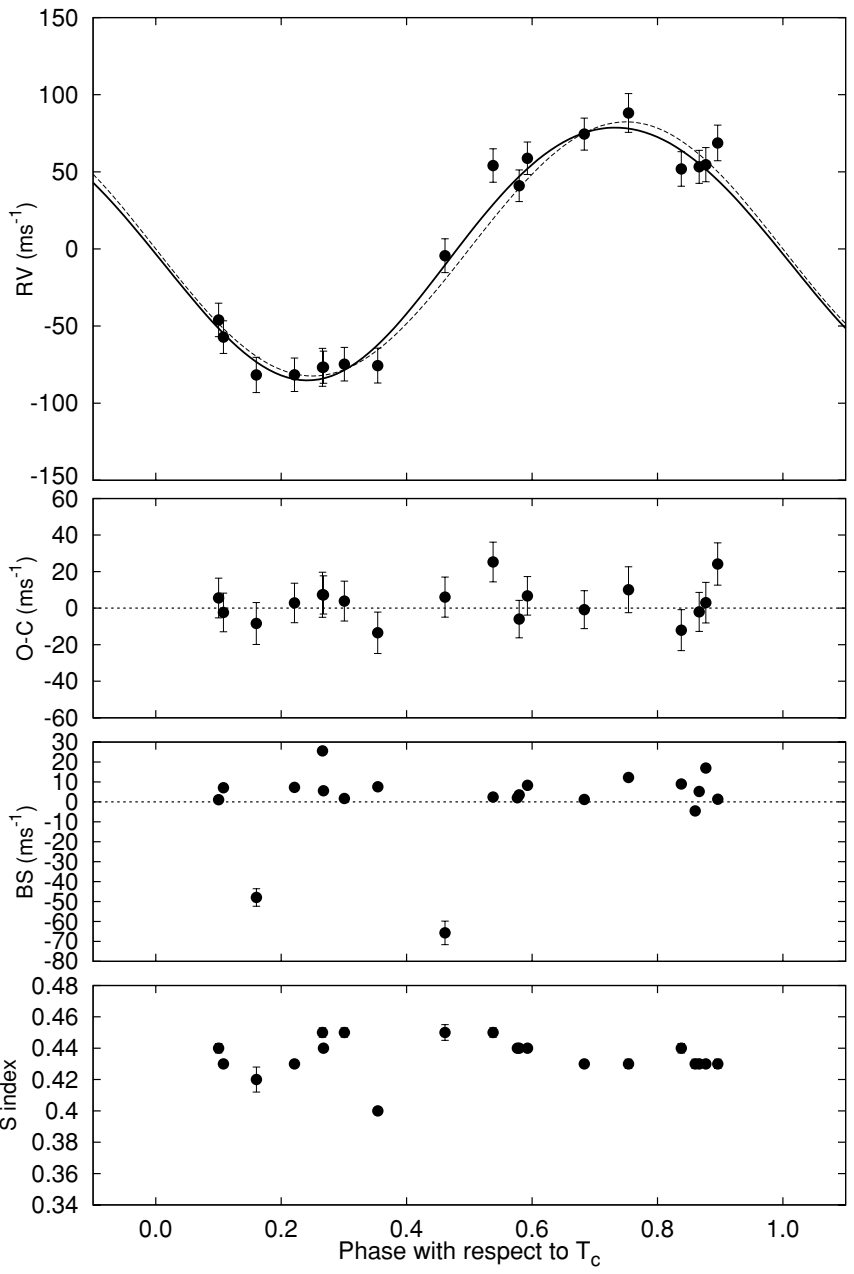

Figure 2. Top panel: Keck/HIRES RV measurements for HAT-P-24 shown as a function of orbital phase, along with our best-fit eccentric orbit model (solid) and circular orbit model (dashed). Zero phase corresponds to the time of mid-transit. The center-of-mass velocity has been subtracted. Second panel: phase-folded velocity $O-C$ residuals from the best fit. The error bars include a component from astrophysical jitter $\left(7.4 \mathrm{~m} \mathrm{~s}^{-1}\right)$ added in quadrature to the formal errors (see Section 3.3). Third panel: BS, with the mean value subtracted. The measurement from the template spectrum is included (see Section 3.2). Bottom panel: relative chromospheric activity index $S$ measured from the Keck spectra. Note the different vertical scales of the panels.

In the same figure, we also show the relative $S$ index, which is a measure of the chromospheric activity of the star derived from the flux in the cores of the $\mathrm{Ca}$ II $\mathrm{H}$ and $\mathrm{K}$ lines. This index was computed following the prescription given by Vaughan et al. (1978), after matching each spectrum to a reference spectrum using a transformation that includes a wavelength shift and a flux scaling that is a polynomial as a function of wavelength. The transformation was determined on regions of the spectra that are not used in computing this indicator. Note that our relative $S$ index has not been calibrated to the scale of Vaughan et al. (1978). The rms of the relative $S$ values is $3 \%$, which is higher than the median formal error of $0.6 \%$ based on photon statistics; however, the errors in this case are likely dominated by systematics in the spectrum matching procedure, which are difficult to quantify, so we do not consider this to be a robust detection of variability. We note that a $3 \%$ variation is comparable to that found for other late F stars (e.g., Shkolnik et al. 2008 measured a $\sim 1 \%$ median absolute deviation in the fluxes of the K line cores for $\tau$ Boo and HD 179949, which corresponds to a similar expected $\mathrm{rms}$ ). 


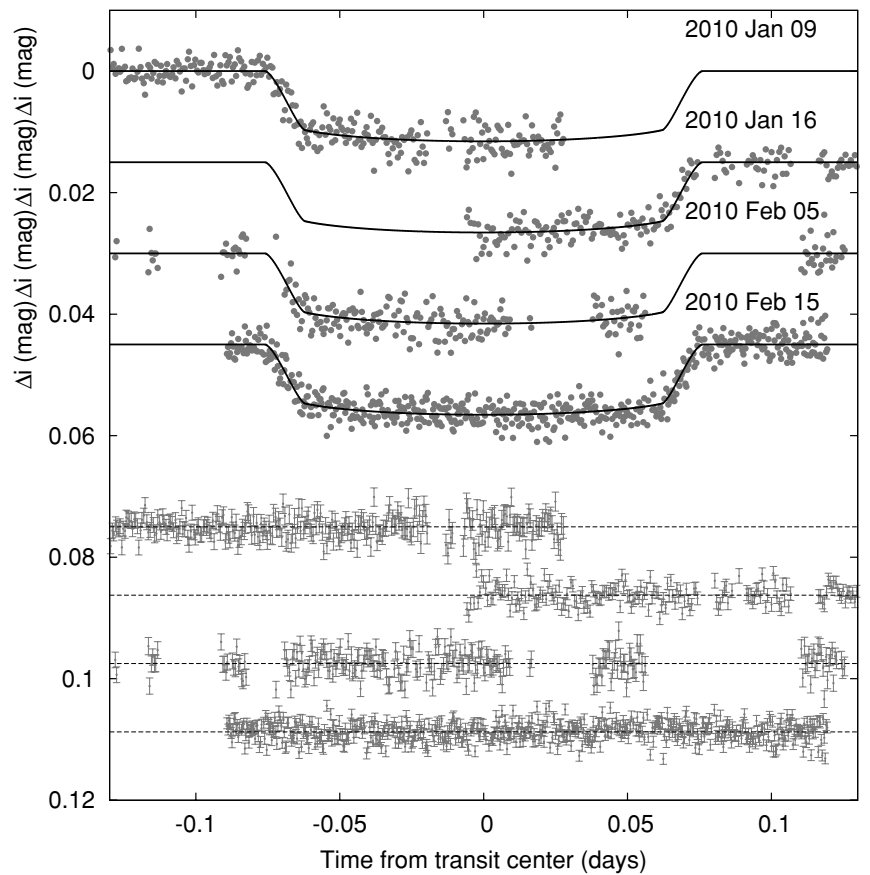

Figure 3. Unbinned normalized Sloan $i$-band transit light curves, acquired with KeplerCam at the FLWO $1.2 \mathrm{~m}$ telescope on 2010 January 9, 2010 January 16, and 2010 February 5, and with the Faulkes Telescope North on 2010 February 15. The light curves have been EPD and TFA processed, as described in Section 3.3. Final fits come from the $\left\{p^{2}, b, T_{1.5,3.5}\right\}$ light-curve parameter set and fitted limb darkening, in combination with an eccentric orbit RV fit. The dates of the events are indicated. Curves after the first are displaced vertically for clarity. Our best fit from the global modeling described in Section 3.3 is shown by the solid lines. Residuals from the fits are displayed at the bottom in the same order as the top curves. The error bars represent the photon and background shot noise, plus the readout noise.

Table 2

Summary of Photometric Follow-up Observations, All of which were Taken in the Sloan $i$ Band

\begin{tabular}{lccc}
\hline \hline Facility & Date & Number of Images & Cadence (s) \\
\hline KeplerCam/FLWO 1.2 m & 2010 Jan 9 & 527 & 39 \\
KeplerCam/FLWO 1.2 m & 2010 Jan 16 & 246 & 53 \\
KeplerCam/FLWO 1.2 m & 2010 Feb 5 & 256 & 39 \\
FTN & 2010 Feb 15 & 534 & 30 \\
\hline
\end{tabular}

We also note that $S$ is uncorrelated with orbital phase; such a correlation might have indicated that the RV variations could be due to stellar activity, casting doubt on the planetary nature of the candidate. There is no sign of emission in the cores of the $\mathrm{Ca}$ II $\mathrm{H}$ and $\mathrm{K}$ lines ( $\mathrm{S} / \mathrm{N} \sim 38$ ) in any of our spectra, from which we conclude that the chromospheric activity level in HAT-P-24 is very low.

\subsection{Photometric Follow-up Observations}

In order to permit a more accurate modeling of the light curve, we conducted additional photometric observations with the KeplerCam CCD camera on the FLWO $1.2 \mathrm{~m}$ telescope in Arizona and with the $2.0 \mathrm{~m}$ Faulkes Telescope North (FTN) at Haleakala Observatory in Hawaii. We observed three transit events of HAT-P-24 with the FLWO $1.2 \mathrm{~m}$ telescope on the nights of 2010 January 9, 2010 January 16, and 2010 February 5 , and a fourth transit event with the FTN on the night of 2010 February 15 (Figure 3). These observations are summarized in Table 2.
Table 3

High-precision Differential Photometry of HAT-P-24

\begin{tabular}{crcc}
\hline \hline $\begin{array}{c}\text { BJD } \\
(2,400,000+)\end{array}$ & \multicolumn{1}{c}{$\mathrm{Mag}^{\mathrm{a}}$} & $\sigma_{\mathrm{Mag}}$ & Filter \\
\hline 55206.63289 & -0.00145 & 0.00176 & $i$ \\
55206.63724 & 0.00220 & 0.00181 & $i$ \\
55206.63790 & -0.00278 & 0.00173 & $i$ \\
55206.64440 & -0.00213 & 0.00174 & $i$ \\
55206.64485 & 0.00093 & 0.00171 & $i$ \\
55206.64548 & -0.00051 & 0.00161 & $i$ \\
55206.64594 & 0.00535 & 0.00161 & $i$ \\
55206.64659 & -0.00391 & 0.00141 & $i$ \\
55206.64703 & 0.00162 & 0.00140 & $i$ \\
55206.64767 & -0.00023 & 0.00134 & $i$ \\
& & &
\end{tabular}

Notes.

a The out-of-transit level has been subtracted. These magnitudes have been subjected to the EPD and TFA procedures, carried out simultaneously with the transit fit.

(This table is available in its entirety in a machine-readable form in the online journal. A portion is shown here for guidance regarding its form and content.)

The reduction of these images, including basic calibration, astrometry, and aperture photometry, was performed as described by Bakos et al. (2010). We performed EPD and TFA to remove trends simultaneously with the light-curve modeling (for more details, see Section 3 and Bakos et al. 2010). The final time series are shown in the top portion of Figure 3, along with our best-fit transit light-curve model described below; the individual measurements are reported in Table 3.

\section{ANALYSIS}

\subsection{Properties of the Parent Star}

Fundamental parameters of the host star HAT-P-24 such as the mass $\left(M_{\star}\right)$ and radius $\left(R_{\star}\right)$, which are needed to infer the planetary properties, depend strongly on other stellar quantities that can be derived spectroscopically. For this, we have relied on our template spectrum obtained with the Keck/HIRES instrument, and the analysis package known as Spectroscopy Made Easy (SME; Valenti \& Piskunov 1996), along with the atomic line database of Valenti \& Fischer (2005). SME yielded the following initial values and uncertainties (which we have conservatively increased for $T_{\text {eff }}$ and $[\mathrm{Fe} / \mathrm{H}]$ to include our estimates of the systematic errors): effective temperature $T_{\mathrm{eff} \star}=$ $6188 \pm 80 \mathrm{~K}$, stellar surface gravity $\log g_{\star}=4.01 \pm 0.06(\mathrm{cgs})$, metallicity $[\mathrm{Fe} / \mathrm{H}]=-0.26 \pm 0.08 \mathrm{dex}$, and projected rotational velocity $v \sin i=10.5 \pm 0.5 \mathrm{~km} \mathrm{~s}^{-1}$.

In principle, the effective temperature and metallicity, along with the surface gravity taken as a luminosity indicator, could be used as constraints to infer the stellar mass and radius by comparison with stellar evolution models. For planetary transits, a stronger constraint is often provided by the $a / R_{\star}-$ normalized semi-major axis, which is closely related to $\rho_{\star}$, the mean stellar density. The quantity $a / R_{\star}$ can be derived directly from the transit light curves (Seager \& Mallén-Ornelas 2003) and the RV data (for eccentric cases, see Kipping 2010). This, in turn, allows us to improve on the determination of the spectroscopic parameters by supplying an indirect constraint on the weakly determined spectroscopic value of $\log g_{\star}$ that removes degeneracies. We take this approach here, as described in Bakos et al. (2010). The validity of our assumption, namely that the adequate physical model describing our data is a 
Table 4

Stellar Parameters for HAT-P-24

\begin{tabular}{lcc}
\hline \hline \multicolumn{1}{c}{ Parameter } & Value & Source \\
\hline Spectroscopic properties & & \\
$T_{\text {eff }}(\mathrm{K})$ & $6373 \pm 80$ & SME $^{\mathrm{a}}$ \\
{$[\mathrm{Fe} / \mathrm{H}]$} & $-0.16 \pm 0.08$ & $\mathrm{SME}$ \\
$v \sin i\left(\mathrm{~km} \mathrm{~s}^{-1}\right)$ & $10.0 \pm 0.5$ & $\mathrm{SME}$ \\
$v_{\text {mac }}\left(\mathrm{km} \mathrm{s}^{-1}\right)$ & 4.94 & $\mathrm{SME}$ \\
$v_{\text {mic }}\left(\mathrm{km} \mathrm{s}^{-1}\right)$ & 0.85 & $\mathrm{SME}$ \\
$\gamma_{\mathrm{RV}}\left(\mathrm{km} \mathrm{s}^{-1}\right)$ & $-2.09 \pm 0.74$ & $\mathrm{DS}$ \\
Photometric properties & & \\
$V(\mathrm{mag})$ & 11.818 & $\mathrm{TASS}$ \\
$V-I_{C}(\mathrm{mag})$ & $0.628 \pm 0.089$ & $\mathrm{TASS}$ \\
$J(\mathrm{mag})$ & $10.797 \pm 0.022$ & $2 \mathrm{MASS}$ \\
$H(\mathrm{mag})$ & $10.589 \pm 0.024$ & $2 \mathrm{MASS}$ \\
$K_{s}(\mathrm{mag})$ & $10.543 \pm 0.020$ & $2 \mathrm{MASS}$ \\
Derived properties & & $\mathrm{YY}+a / R_{\star}+\mathrm{SME}$ \\
$M_{\star}\left(M_{\odot}\right)$ & $1.191 \pm 0.042$ & $\mathrm{YY}+a / R_{\star}+\mathrm{SME}$ \\
$R_{\star}\left(R_{\odot}\right)$ & $1.317 \pm 0.068$ & $\mathrm{YY}+a / R_{\star}+\mathrm{SME}$ \\
$\log g_{\star}(\mathrm{cgs})$ & $4.27 \pm 0.04$ & $\mathrm{YY}+a / R_{\star}+\mathrm{SME}$ \\
$L_{\star}\left(L_{\odot}\right)$ & $2.56 \pm 0.31$ & $\mathrm{YY}+a / R_{\star}+\mathrm{SME}$ \\
$M_{V}(\mathrm{mag})$ & $3.74 \pm 0.14$ & $\mathrm{YY}+a / R_{\star}+\mathrm{SME}$ \\
$M_{K}(\mathrm{mag}, \mathrm{ESO})$ & $2.59 \pm 0.11$ & $\mathrm{YY}+a / R_{\star}+\mathrm{SME}$ \\
Age $(\mathrm{Gyr})$ & $2.8 \pm 0.6$ & \\
Distance $(\mathrm{pc})$ & $396 \pm 20 / R_{\star}+\mathrm{SME}$ \\
\hline
\end{tabular}

Notes.

a SME = "Spectroscopy Made Easy" package for the analysis of high-resolution spectra (Valenti \& Piskunov 1996). These parameters rely primarily on SME, but have a small dependence also on the iterative analysis incorporating the isochrone search and global modeling of the data, as described in the text.

b $\mathrm{YY}+a / R_{\star}+\mathrm{SME}=$ based on the $\mathrm{YY}$ isochrones (Yi et al. 2001), $a / R_{\star}$ as a luminosity indicator, and the SME results.

planetary transit (as opposed to a blend), is shown later in Section 3.2.

After the first iteration for determining the stellar properties, as described in Bakos et al. (2010), we find that the surface gravity, $\log g_{\star}=4.27 \pm 0.04$, is significantly different from our initial SME analysis, which is not surprising in view of the strong correlations among $T_{\mathrm{eff} \star},[\mathrm{Fe} / \mathrm{H}]$, and $\log g_{\star}$ that are often present in spectroscopic determinations. Therefore, we carried out a second iteration in which we adopted this value of $\log g_{\star}$ and held it fixed in a new SME analysis (coupled with a new global modeling of the RV and light curves), adjusting only $T_{\mathrm{eff} \star},[\mathrm{Fe} / \mathrm{H}]$, and $v \sin i$. This gave $T_{\mathrm{eff} \star}=6373 \pm 80 \mathrm{~K}$, $[\mathrm{Fe} / \mathrm{H}]=-0.16 \pm 0.08$, and $v \sin i=10.0 \pm 0.5 \mathrm{~km} \mathrm{~s}^{-1}$, in which the conservative uncertainties for the first two have been increased by a factor of 2 over their formal values, as before. Experience with the SME analysis for previous HAT planets leads us to select this estimate. A further iteration did not change $\log g_{\star}$ significantly, so we adopted the values stated above as the final atmospheric properties of the star. They are collected in Table 4, together with the adopted values for the macroturbulent and microturbulent velocities.

The low metallicity of HAT-P-24 is interesting in that a wellknown bias exists for finding giant planets around metal-rich stars (Johnson et al. 2010). Johnson et al. (2010) find that the occurrence of giant planets scales as $f \sim 10^{1.2[\mathrm{Fe} / \mathrm{H}]}$ and also report a scaling with stellar mass of $f \sim M_{*}$. This means that the a priori probability of finding a planet around HAT-P-24 is a respectable $\sim 75 \%$ that of a solar-like star.

With the adopted spectroscopic parameters, the model isochrones yield the stellar mass and radius $M_{\star}=1.191 \pm$ $0.042 M_{\odot}$ and $R_{\star}=1.317 \pm 0.068 R_{\odot}$, along with other proper-

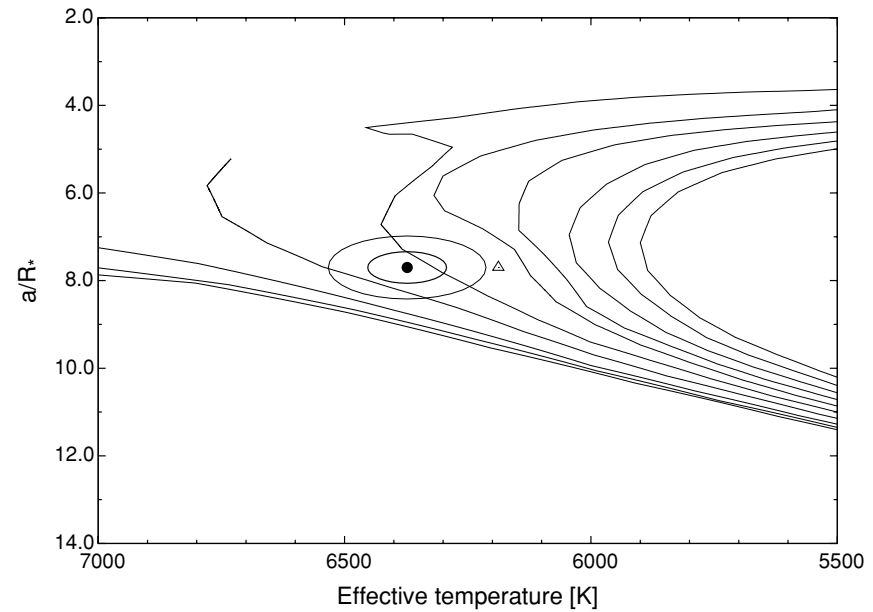

Figure 4. Model isochrones from Yi et al. (2001) for the measured metallicity of HAT-P-24, $[\mathrm{Fe} / \mathrm{H}]=-0.16$, and ages of $0.2,0.5,1.0,2.0,3.0,4.0,5.0$, 6.0, 7.0, and 8.0 Gyr (left to right). The adopted values of $T_{\mathrm{eff} \star}$ and $a / R_{\star}$ are shown together with their $1 \sigma$ and $2 \sigma$ confidence ellipsoids. The results come from the $\left\{p^{2}, b, T_{1.5,3.5}\right\}$ light-curve parameter set and fitted limb darkening, in combination with an eccentric orbit RV fit. The initial values of $T_{\operatorname{eff} \star}$ and $a / R_{\star}$ from the first SME and light-curve analyses are represented with a triangle.

ties listed at the bottom of Table 4. HAT-P-24 is an F8 dwarf star with an estimated age of $2.8 \pm 0.6 \mathrm{Gyr}$, according to these models (Yi et al. 2001). The inferred location of the star in a diagram of $a / R_{\star}$ versus $T_{\text {eff } \star}$, analogous to the classical H-R diagram, is shown in Figure 4 . The stellar properties and their $1 \sigma$ and $2 \sigma$ confidence ellipsoids are displayed against the backdrop of $\mathrm{Yi}$ et al. (2001) isochrones for the measured metallicity of $[\mathrm{Fe} / \mathrm{H}]$ $=-0.16$, and a range of ages. For comparison, the location implied by the initial SME results is also shown (triangle) and corresponds to a somewhat more evolved state.

The stellar evolution modeling provides color indices that may be compared against the measured values as a consistency check. The best available measurements are the near-infrared magnitudes from the 2MASS Catalog (Skrutskie et al. 2006), $J_{\text {MASS }}=10.797 \pm 0.022, H_{2 \mathrm{MASS}}=10.589 \pm 0.024$, and $K_{2 \mathrm{MASS}}=10.543 \pm 0.020$, which we have converted to the photometric system of the models (ESO system) using the transformations by Carpenter (2001). The resulting measured color index is $J-K=0.274 \pm 0.032$. This is within $1 \sigma$ of the predicted value from the isochrones of $J-K=0.29 \pm 0.02$. The distance to the object may be computed from the absolute $K$ magnitude from the models $\left(M_{\mathrm{K}}=2.59 \pm 0.11\right)$ and the 2MASS $K_{s}$ magnitude, which has the advantage of being less affected by extinction than optical magnitudes. The result is $396 \pm 20 \mathrm{pc}$, where the uncertainty excludes possible systematics in the model isochrones that are difficult to quantify.

\subsection{Spectral Line-bisector Analysis}

Our initial spectroscopic analyses discussed in Sections 2.2 and 2.3 rule out the most obvious astrophysical false positive scenarios. However, more subtle phenomena such as blends (contamination by an unresolved eclipsing binary, whether in the background or associated with the target) can still mimic both the photometric and spectroscopic signatures we see.

Following Torres et al. (2007), we explored the possibility that the measured RVs are not real, but are instead caused by distortions in the spectral line profiles due to contamination from a nearby unresolved eclipsing binary (Queloz et al. 2001). A bisector analysis based on the Keck spectra was done as 
described in Section 5 of Bakos et al. (2007). We detect no correlated variation between the bisector spans and the RVs (see Figure 2). All but two of the bisector measurements are consistent with no variation. Following the methods described in Hartman et al. (2009) and Kovács et al. (2010), we estimated the expected effect of contamination from scattered moonlight on the bisectors, finding that the two outlier measurements correspond to the two spectra that are expected to be the most affected by sky contamination, when the Moon is $70^{\circ}$ from the target. Therefore, we conclude that the velocity variations are real, and that the star is orbited by a close-in giant planet.

\subsection{Global Modeling of the Data}

This section briefly describes the procedure we followed to model the HATNet photometry, the follow-up photometry, and the RVs simultaneously. More details on the fitting methods can be found in Bakos et al. (2010). Our model for the follow-up light curves used the analytic formulae of Mandel \& Agol (2002) with quadratic limb-darkening coefficients interpolated from the tables by Claret (2004). The transit shape was parameterized by the ratio of radii $p \equiv R_{p} / R_{\star}$, the square of the impact parameter $b^{2}$, and the reciprocal of the half duration of the transit $\zeta / R_{\star}$. We denote this fitting set as $\left\{p, b^{2}, \zeta / R_{*}\right\}$. This set is chosen because of their simple geometric meanings and the fact that these exhibit low correlations (see Bakos et al. 2010). Our model for the HATNet data was the simplified "P1P3" version of the Mandel \& Agol (2002) analytic functions following the method of Bakos et al. (2010). Following the formalism presented by Pál (2009a), the RVs were fitted with an eccentric Keplerian model parameterized by the semi-amplitude $K$ and Lagrangian elements $k \equiv e \cos \omega$ and $h \equiv e \sin \omega$, in which $\omega$ is the longitude of periastron.

Assuming a linear ephemeris, we assign the transit number $N_{\text {tr }}=0$ to the complete follow-up light curve gathered on 2010 February 15 . The eight main parameters describing the physical model were thus $T_{c,-250}, T_{c, 0}, R_{p} / R_{\star}, b^{2}, \zeta / R_{\star}, K, k \equiv e \cos \omega$, and $h \equiv e \sin \omega$. Five additional parameters were included that have to do with the instrumental configuration. These are the HATNet blend factors $B_{\text {inst. } 315}$ and $B_{\text {inst. } 316}$, which account for possible dilution of the transit in the HATNet light curves from background stars due to the broad point-spread function $\left(20^{\prime \prime} \mathrm{FWHM}\right)$, the HATNet out-of-transit magnitudes $M_{0, \text { HATNet, } 315}$ and $M_{0, \text { HATNet,316 }}$, and the relative zero point $\gamma_{\text {rel }}$ of the Keck RVs.

We extended our physical model with an instrumental model that describes brightness variations caused by systematic errors in the measurements as described in Bakos et al. (2010). The HATNet photometry has already been EPD- and TFA-corrected before the global modeling, so we only considered corrections for systematics in the follow-up light curves. We chose the "ELTG" method, i.e., EPD was performed in the "local" mode with EPD coefficients defined for each night, and TFA was performed in the "global" mode using the same set of stars and TFA coefficients for all nights, as done in Bakos et al. (2010).

The joint fit was accomplished using downhill simplex (AMOEBA; see Press et al. 1992) and the Markov Chain Monte Carlo method (MCMC; see Ford 2006) using "HyperplaneCLLS" chains (Bakos et al. 2010) and the analytic partial derivatives for the transit light curve from Pál (2009a). A detailed description can be found in Bakos et al. (2010). The resulting geometric parameters pertaining to the light curves and velocity curves are listed in Table 5. Quoted values are the median and
Table 5

Orbital and Planetary Parameters

\begin{tabular}{|c|c|}
\hline Parameter & Value \\
\hline \multicolumn{2}{|l|}{ Light-curve parameters } \\
\hline$P$ (days) & $3.355240 \pm 0.000007$ \\
\hline$T_{c}(\mathrm{BJD})^{\mathrm{a}}$ & $2455216.97667 \pm 0.00028$ \\
\hline$T_{14}(\text { days })^{\mathrm{a}}$ & $0.1539 \pm 0.0008$ \\
\hline$T_{12}=T_{34}(\text { days })^{\mathrm{a}}$ & $0.0141 \pm 0.0006$ \\
\hline$a / R_{\star}$ & $7.58 \pm 0.35$ \\
\hline$\zeta / R_{\star}$ & $14.31 \pm 0.06$ \\
\hline$R_{p} / R_{\star}$ & $0.0970 \pm 0.0012$ \\
\hline$b^{2}$ & $0.036^{+0.042}$ \\
\hline$b \equiv a \cos i / R_{\star}$ & $0.189_{-0.080}^{+0.0821}$ \\
\hline$i(\mathrm{deg})$ & $88.6 \pm 0.7$ \\
\hline \multicolumn{2}{|l|}{ Limb-darkening coefficients ${ }^{b}$} \\
\hline$a_{i}($ linear term) & 0.1858 \\
\hline$b_{i}$ (quadratic term) & 0.3625 \\
\hline \multicolumn{2}{|l|}{ RV parameters } \\
\hline$K\left(\mathrm{~m} \mathrm{~s}^{-1}\right)$ & $83.0 \pm 3.4$ \\
\hline$k_{\mathrm{RV}}^{\mathrm{c}}$ & $-0.053 \pm 0.021$ \\
\hline$h_{\mathrm{RV}}{ }^{\mathrm{c}}$ & $-0.017 \pm 0.042$ \\
\hline$e$ & $0.067 \pm 0.024$ \\
\hline$\omega(\mathrm{deg})$ & $197^{\circ} \pm 36$ \\
\hline $\mathrm{RV}$ jitter $\left(\mathrm{m} \mathrm{s}^{-1}\right)$ & 7.4 \\
\hline \multicolumn{2}{|l|}{ Secondary eclipse parameters } \\
\hline$T_{s}(\mathrm{BJD})$ & $2455218.542 \pm 0.045$ \\
\hline$T_{S, 14}$ & $0.1492 \pm 0.0121$ \\
\hline$T_{s, 12}$ & $0.0137 \pm 0.0013$ \\
\hline \multicolumn{2}{|l|}{$\begin{array}{l}1_{S, 12} \\
\text { Planetary parameters }\end{array}$} \\
\hline$M_{p}\left(M_{\mathrm{J}}\right)$ & $0.685 \pm 0.033$ \\
\hline$R_{p}\left(R_{\mathrm{J}}\right)$ & $1.242 \pm 0.067$ \\
\hline$C\left(M_{p}, R_{p}\right)^{\mathrm{d}}$ & 0.31 \\
\hline$\rho_{p}\left(\mathrm{~g} \mathrm{~cm}^{-3}\right)$ & $0.44 \pm 0.07$ \\
\hline $\log g_{p}(\mathrm{cgs})$ & $3.04 \pm 0.05$ \\
\hline$a(\mathrm{AU})$ & $0.0465 \pm 0.0006$ \\
\hline$T_{\mathrm{eq}}(\mathrm{K})$ & $1637 \pm 42$ \\
\hline$\Theta^{e^{(-1)}}$ & $0.043 \pm 0.003$ \\
\hline$F_{\text {per }}\left(10^{9} \mathrm{erg} \mathrm{s}^{-1} \mathrm{~cm}^{-2}\right)$ & $1.84 \pm 0.208$ \\
\hline$F_{\text {ap }}\left(10^{9} \mathrm{erg} \mathrm{s}^{-1} \mathrm{~cm}^{-2}\right)$ & $1.44 \pm 0.168$ \\
\hline$\langle F\rangle\left(10^{9} \mathrm{erg} \mathrm{s}^{-1} \mathrm{~cm}^{-2}\right)^{\mathrm{f}}$ & $1.62 \pm 0.169$ \\
\hline
\end{tabular}

Notes.

${ }^{\text {a }} T_{c}$ : Reference epoch of mid transit that minimizes the correlation with the orbital period. It corresponds to $N_{\mathrm{tr}}=-8$. BJD is calculated from UTC. $T_{14}$ : total transit duration. $T_{12}=T_{34}$ : ingress/egress time.

b Values for a quadratic law, adopted from the tabulations by Claret (2004) according to the spectroscopic (SME) parameters listed in Table 4.

${ }^{\mathrm{c}}$ Lagrangian orbital parameters derived from the global modeling, and primarily determined by the RV data.

${ }^{\mathrm{d}}$ Correlation coefficient between the planetary mass $M_{p}$ and radius $R_{p}$.

e The Safronov number is given by $\Theta=\frac{1}{2}\left(V_{\mathrm{esc}} / V_{\mathrm{orb}}\right)^{2}=\left(a / R_{p}\right)\left(M_{p} / M_{\star}\right)$ (see Hansen \& Barman 2007).

${ }^{\mathrm{f}}$ Incoming flux per unit surface area, averaged over the orbit.

the error on the median from the a posteriori distribution of each parameter.

Included in this table is the RV "jitter," which we added in quadrature to the internal errors for the RVs in order to achieve $\chi^{2} /$ dof $=1$ from the RV data for the global fit. Auxiliary parameters not listed in the table are: $T_{\mathrm{c},-250}=$ $2454405.00857 \pm 0.00162(\mathrm{BJD}), T_{\mathrm{c}, 0}=2455243.81859 \pm$ 0.00029 (BJD), the blending factors $B_{\text {instr } 314}=0.83 \pm 0.05$ and $B_{\text {instr } 315}=0.69 \pm 0.03$, and $\gamma_{\text {rel }}=3.20 \pm 2.62 \mathrm{~m} \mathrm{~s}^{-1}$. The latter quantity represents an arbitrary offset for the Keck RVs, and does not correspond to the true center of mass velocity of the system, which was listed earlier in Table $4\left(\gamma_{\mathrm{RV}}\right)$. 
The planetary parameters and their uncertainties can be derived by combining the a posteriori distributions for the stellar, light curve, and RV parameters. In this way, we find a mass for the planet of $M_{p}=0.685 \pm 0.033 M_{\mathrm{J}}$ and a radius of $R_{p}=1.242 \pm 0.067 R_{\mathrm{J}}$, leading to a mean density $\rho_{p}=0.44 \pm 0.07 \mathrm{~g} \mathrm{~cm}^{-3}$. These and other planetary parameters are listed at the bottom of Table 5. We note that the system may be slightly eccentric: $e=0.067 \pm 0.024, \omega=197^{\circ} \pm 36^{\circ}$.

\section{COMPARISON OF FITTING METHODS}

\subsection{Alternative Fitting Parameter Sets}

The fitting method adopted for the values quoted in Table 5 uses the parameter set $\left\{p, \zeta / R_{*}, b^{2}\right\}$, chosen for their low interparameter correlations. $\zeta / R_{*}$ is the reciprocal of the halfduration as computed using an approximate expression for the duration coming from Tingley \& Sackett (2005). Recently, Kipping (2010) showed that an improved approximate formula is possible for the duration. Replacing $\zeta / R_{*}$ with the reciprocal of the new expression for the half-duration therefore offers greater accuracy for the duration determination. Kipping (2010) labeled this parameter as $\Upsilon / R_{*}$ to be distinct from $\zeta / R_{*}$. The new parameter set yields the greatest improvements for neargrazing, low-eccentricity orbits.

To investigate the robustness of the results against different parameter sets, we refitted the EPD, TFA-corrected HAT, and FLWO light curves in conjunction with the RVs using the $\left\{p^{2}, b^{2}, \Upsilon / R_{*}\right\}$ parameter set. RVs are fitted using the Lagrange parameters as in Section 3.3. A new YY (Yi et al. 2001) isochrone analysis is performed as described in Section 3.1 to show the effect on the physical parameters. The results are shown in the first results column of Table 6.

The $b^{2}$ parameter also gives us some pause for thought. One natural reason to select $b^{2}$ is that the duration of a transit is completely described in terms of $b^{2}$, i.e., there is no case of an isolated $b$ which is not squared in the expression for the duration. Therefore, $b^{2}$ seems to be a natural parameter of the transit light curve. Furthermore, $b^{2}$ usually shows lower interparameter correlations than $b$.

However, there are also two reasons why one should not choose $b^{2}$. First, geometrically $b$ is more likely to have a uniform prior than $b^{2}$. Therefore, low signal-to-noise transits will be biased toward higher impact parameters by fitting for $b^{2}$. Second, $b^{2}$ cannot be negative and thus the posterior distribution of $b^{2}$ tends to get offset to an artificially more positive value due to the boundary condition that $b^{2}>0$. One resolution to this is to use $b$ and let the parameter explore both negative and positive values. Whilst a negative impact parameter may seem unphysical, the issue of its physicality is also irrelevant since the transit is completely described in terms of $b^{2}$ and thus a negative $b$ is always multiplied by itself when computing the light-curve morphology. Using $b$ in this way permits for a symmetric distribution about $b=0$ and thus improved estimates of the associated uncertainty.

To investigate the value of fitting for $b$, we repeated our global fits using the parameter set $\left\{p^{2}, b, T_{1.5,3.5}\right\}$. $T_{1.5,3.5}$ replaces $\Upsilon / R_{*}$ since although it may be slightly more correlated, it is more reasonable to expect a uniform prior on the duration than a uniform prior on its reciprocal. The results of these fits are shown in the third results column of Table 6.

The system values quoted between $\left\{p^{2}, b^{2}, \zeta / R_{*}\right\},\left\{p^{2}\right.$, $\left.b^{2}, \Upsilon / R_{*}\right\}$, and $\left\{p^{2}, b, T_{1.5,3.5}\right\}$ show excellent agreement and thus indicate that the system parameters are insensitive to the choice of priors.

\subsection{Fitted Limb Darkening}

We repeated the fits for the $\left\{p^{2}, b^{2}, \Upsilon / R_{*}\right\}$ and $\left\{p^{2}, b, T_{1.5,3.5}\right\}$ parameter sets with fitted limb darkening (second and fourth result columns of Table 6 , respectively). In order to achieve convergence, we choose to only use linear limb darkening and thus fix the quadratic coefficient to be zero.

The results show slight differences with the fixed-limbdarkening analogues. The most noticeable effect is increased error bars. Fitting for limb darkening means that our results are no longer dependent upon a stellar atmosphere model prediction, which is a clear desideratum. Our preferred final values are given by the $\left\{p^{2}, b, T_{1.5,3.5}\right\}$ parameter set with fitted limb darkening (last column of Table 6, with highlighted header).

Both free limb-darkening fits converge at $u_{1}=0.25 \pm 0.04$. For $0.8<\mu<1$, the limb darkening from both the theoretical quadratic coefficients and the fitted coefficients is approximately equivalent. However, for low $\mu$, the theoretical coefficients predict much stronger darkening effects than observed. Fitting a linear law through the quadratic coefficients gives $u_{\text {lin }}=0.46$, demonstrating the stronger limb darkening predicted from theory.

\section{ORBITAL ECCENTRICITY}

\subsection{Significance of the Eccentric Fit}

The eccentric fit suggests an orbital eccentricity of $e=$ $0.052_{-0.017}^{+0.022}$. In addition to the four fits displayed in Table 6, we repeated our preferred model fit (i.e., $\left.p^{2}, b, T_{1.5,3.5}\right\}$ with fitted limb darkening) for a circular orbit. This was done to provide a $\chi^{2}$ value for both fits, which can be used to infer the statistical significance of the eccentric fit.

To accomplish this, we only take the $\chi^{2}$ from the RV data, which dominates the determination of the Lagrangian orbital parameters and so the number of data points is $n=18$. The period and time of transit are dominated by the photometry and very weakly affected by the few RV points. Therefore, these two degrees of freedom can be considered fixed. This leaves us with four degrees of freedom for an eccentric fit $(k, h, K, \gamma)$ and two for the circular fit $(K, \gamma)$.

In evaluating the significance of the eccentric fit over the circular model it is important to penalize the eccentric fit for using two extra degrees of freedom. We therefore choose to perform an F-test between the two models. The circular orbit fit has $\chi^{2}=59.5$ and the eccentric fit has $\chi^{2}=39.6$. The false alarm probability from an F-test is evaluated to be $5.8 \%$ or $1.9 \sigma$. We also performed the test of Lucy \& Sweeney (1971), where the statistical significance of the eccentric fit is given by

$$
P(e>0)=1-\exp \left[-\frac{\hat{e}^{2}}{2 \sigma_{e}^{2}}\right],
$$

where $\hat{e}$ is the modal value of the eccentricity, which is well approximated by the median for a unimode distribution. Using the Lucy \& Sweeney (1971) test, we find an eccentric fit is accepted at the $2.6 \sigma$ level. The slightly higher significance likely comes from the fact this test does not penalize an eccentric model for using more degrees of freedom, whereas the F-test does. Therefore, based upon the current data for this system, an eccentric fit is probable but not conclusive. 
Table 6

Orbital and Planetary Parameters

\begin{tabular}{|c|c|c|c|c|}
\hline Parameter & $\left\{b^{2}, \Upsilon / R_{*}\right\}$, fixed LD & $\left\{b^{2}, \Upsilon / R_{*}\right\}$, fitted LD & $\left\{b, T_{1.5,3.5}\right\}$, fixed LD & $\left\{\mathbf{b}, \mathbf{T}_{1.5,3.5}\right\}$, fitted LD \\
\hline \multicolumn{5}{|l|}{ Light curve parameters } \\
\hline$P$ (days) & $3.3552459_{-0.0000072}^{+0.0000079}$ & $3.3552466_{-0.0000070}^{+0.0000069}$ & $3.3552458_{-0.0000072}^{+0.0000078}$ & $3.3552464_{-0.0000071}^{+0.000069}$ \\
\hline$T_{c}\left(\mathrm{BJD}_{\mathrm{UTC}}-2,450,000\right)^{\mathrm{a}}$ & $5216.97672_{-0.00025}^{+0.00025}$ & $5216.97668_{-0.00024}^{+0.00024}$ & $5216.97672_{-0.00025}^{+0.00024}$ & $5216.97669_{-0.00024}^{+0.00024}$ \\
\hline$T_{1,4}(\mathrm{~s})^{\mathrm{a}}$ & $13307.3_{-55.2}^{+62.0}$ & $13172.1_{-79.3}^{+100.5}$ & $13307.8_{-53.8}^{+58.7}$ & $13152.7_{-69.2}^{+88.6}$ \\
\hline$T_{1.5,3.5}(\mathrm{~s})^{\mathrm{a}}$ & $12112.1_{-44.5}^{+44.0}$ & $11908.8_{-52.6}^{+53.4}$ & $12111.8_{-44.6}^{+45.1}$ & $11912.1_{-52.7}^{+52.7}$ \\
\hline$T_{2,3}(\mathrm{~s})^{\mathrm{a}}$ & $10917.0_{-53.9}^{+46.3}$ & $10646.4_{-139.6}^{+97.2}$ & $10915.8_{-49.0}^{+45.1}$ & $10676.6_{-121.0}^{+75.7}$ \\
\hline$T_{1,2} \simeq T_{3,4}(\mathrm{~s})^{\mathrm{a}}$ & $1184.4_{-11.3}^{+47.2}$ & $1259.5_{-75.6}^{+110.8}$ & $1189.3_{-12.9}^{+32.3}$ & $1227.4_{-41.9}^{+101.3}$ \\
\hline$\left(R_{P} / R_{*}\right)^{2}(\%)$ & $0.9363_{-0.0092}^{+0.0093}$ & $0.978_{-0.014}^{+0.015}$ & $0.9362_{-0.0090}^{+0.0091}$ & $0.976_{-0.013}^{+0.014}$ \\
\hline$R_{P} / R_{*}$ & $0.09676_{-0.00047}^{+0.00048}$ & $0.09889_{-0.00071}^{+0.00073}$ & $0.09676_{-0.00047}^{+0.00047}$ & $0.09877_{-0.00068}^{+0.00069}$ \\
\hline$a / R_{*}$ & $7.71_{-0.31}^{+0.32}$ & $7.63_{-0.38}^{+0.37}$ & $7.71_{-0.30}^{+0.31}$ & $7.70_{-0.36}^{+0.35}$ \\
\hline$\Upsilon / R_{*}\left(\right.$ days $\left.^{-1}\right)$ & $14.267_{-0.052}^{+0.053}$ & $14.510_{-0.065}^{+0.064}$ & $14.267_{-0.053}^{+0.053}$ & $14.506_{-0.064}^{+0.064}$ \\
\hline$b$ & $0.00_{-0.00}^{+0.20}$ & $0.25_{-0.24}^{+0.12}$ & $0.00_{-0.13}^{+0.13}$ & $0.00_{-0.26}^{+0.26}$ \\
\hline$b^{2}$ & $0.000_{-0.000}^{+0.041}$ & $0.060_{-0.060}^{+0.072}$ & $0.008_{-0.007}^{+0.025}$ & $\mathbf{0 . 0 3 7} 7_{-0.033}^{+0.070}$ \\
\hline$i(\mathrm{deg})$ & $90.0_{-1.5}^{+0.0}$ & $88.2_{-1.0}^{+1.8}$ & $90.0_{-1.0}^{+1.0}$ & $90.0_{-1.9}^{+1.9}$ \\
\hline$\rho_{*}\left(\mathrm{~g} \mathrm{~cm}^{-3}\right)$ & $0.770_{-0.088}^{+0.099}$ & $0.748_{-0.105}^{+0.114}$ & $0.771_{-0.086}^{+0.097}$ & $0.768_{-0.102}^{+0.110}$ \\
\hline \multicolumn{5}{|l|}{ Limb-darkening coefficients ${ }^{b}$} \\
\hline$u_{1}($ linear term) & $0.1858^{*}$ & $0.249_{-0.039}^{+0.038}$ & $0.1858^{*}$ & $0.251_{-0.038}^{+0.037}$ \\
\hline$u_{2}$ (quadratic term) & $0.3625^{*}$ & $0^{*}$ & $0.3625^{*}$ & $\mathbf{0}^{*}$ \\
\hline \multicolumn{5}{|l|}{ RV derived parameters } \\
\hline$k_{\mathrm{RV}}^{\mathrm{c}}$ & $-0.037_{-0.014}^{+0.014}$ & $-0.037_{-0.014}^{+0.014}$ & $-0.037_{-0.014}^{+0.014}$ & $-0.037_{-0.014}^{+0.014}$ \\
\hline$h_{\mathrm{RV}}^{\mathrm{c}}$ & $-0.018_{-0.039}^{+0.038}$ & $-0.017_{-0.039}^{+0.038}$ & $-0.018_{-0.038}^{+0.038}$ & $-0.018_{-0.039}^{+0.038}$ \\
\hline$\Psi^{\mathrm{c}}$ & $1.007_{-0.004}^{+0.012}$ & $1.006_{-0.004}^{+0.012}$ & $1.006_{-0.004}^{+0.012}$ & $1.007_{-0.004}^{+0.012}$ \\
\hline$K\left(\mathrm{~m} \mathrm{~s}^{-1}\right)$ & $82.6_{-3.2}^{+3.1}$ & $82.6_{-3.2}^{+3.1}$ & $82.6_{-3.1}^{+3.1}$ & $82.6_{-3.1}^{+3.2}$ \\
\hline$e$ & $0.052_{-0.017}^{+0.022}$ & $0.052_{-0.017}^{+0.022}$ & $0.052_{-0.017}^{+0.022}$ & $\mathbf{0 . 0 5 2} 2_{-0.017}^{+0.022}$ \\
\hline$\omega(\operatorname{deg})$ & $206_{-54}^{+35}$ & $205_{-54}^{+36}$ & $206_{-54}^{+35}$ & $206_{-53}^{+36}$ \\
\hline $\mathrm{RV}$ jitter $\left(\mathrm{m} \mathrm{s}^{-1}\right)$ & $7.53_{-7.53}^{+3.60}$ & $7.44_{-7.44}^{+3.54}$ & $7.58_{-7.58}^{+3.65}$ & $\mathbf{7 . 4 3}+\mathbf{7 . 4 3}$ \\
\hline $\log g_{p}(\mathrm{cgs})$ & $3.055_{-0.040}^{+0.039}$ & $3.027_{-0.050}^{+0.046}$ & $3.055_{-0.038}^{+0.039}$ & $3.037_{-0.047}^{+0.043}$ \\
\hline \multicolumn{5}{|l|}{ Secondary eclipse parameters } \\
\hline$T_{S}\left(\mathrm{BJD}_{\mathrm{UTC}}-2,450,000\right)$ & $5218.575_{-0.030}^{+0.030}$ & $55218.575_{-0.030}^{+0.030}$ & $5218.576_{-0.030}^{+0.029}$ & $\mathbf{5 2 1 8 . 5 7 5}_{-0.030}^{+0.030}$ \\
\hline$T_{S, 1,4}(\mathrm{~s})$ & $12850_{-940}^{+1000}$ & $12770_{-910}^{+930}$ & $12850_{-940}^{+990}$ & $12730_{-920}^{+950}$ \\
\hline \multicolumn{5}{|l|}{ Stellar parameters } \\
\hline$M_{*}\left(M_{\odot}\right)$ & $1.184_{-0.039}^{+0.041}$ & $1.189_{-0.041}^{+0.041}$ & $1.184_{-0.039}^{+0.040}$ & $1.186_{-0.041}^{+0.042}$ \\
\hline$R_{*}\left(R_{\odot}\right)$ & $1.293_{-0.057}^{+0.061}$ & $1.307_{-0.066}^{+0.077}$ & $1.293_{-0.056}^{+0.058}$ & $1.294_{-0.062}^{+0.071}$ \\
\hline $\log \left(g_{*}\right)(\mathrm{cgs})$ & $4.287_{-0.034}^{+0.034}$ & $4.279_{-0.042}^{+0.040}$ & $4.287_{-0.033}^{+0.034}$ & $4.286_{-0.039}^{+0.038}$ \\
\hline$L_{*}\left(L_{\odot}\right)$ & $2.47_{-0.26}^{+0.29}$ & $2.52_{-0.29}^{+0.35}$ & $2.47_{-0.25}^{+0.28}$ & $2.48_{-0.28}^{+0.32}$ \\
\hline$M_{V}(\mathrm{mag})$ & $3.78_{-0.13}^{+0.13}$ & $3.76_{-0.14}^{+0.14}$ & $3.78_{-0.12}^{+0.13}$ & $3.78_{-0.14}^{+0.14}$ \\
\hline Age (Gyr) & $2.75_{-0.64}^{+0.58}$ & $2.80_{-0.63}^{+0.55}$ & $2.75_{-0.64}^{+0.58}$ & $2.75_{-0.65}^{+0.58}$ \\
\hline Distance (pc) & $404_{-23}^{+24}$ & $409_{-26}^{+28}$ & $404_{-23}^{+24}$ & $405_{-25}^{+27}$ \\
\hline \multicolumn{5}{|l|}{ Planetary parameters } \\
\hline$M_{P}\left(M_{J}\right)$ & $0.680_{-0.030}^{+0.030}$ & $0.682_{-0.031}^{+0.031}$ & $0.680_{-0.030}^{+0.030}$ & $0.681_{-0.030}^{+0.031}$ \\
\hline$R_{P}\left(R_{J}\right)$ & $1.217_{-0.054}^{+0.0581}$ & $1.257_{-0.066}^{+0.079}$ & $1.217_{-0.053}^{+0.056}$ & $1.243_{-0.061}^{+0.072}$ \\
\hline $\mathrm{C}\left\{M_{P} / R_{P}\right\}^{\mathrm{d}}$ & 0.264 & 0.300 & 0.266 & 0.290 \\
\hline$\rho_{P}\left(\mathrm{~g} \mathrm{~cm}^{-3}\right)$ & $0.467_{-0.059}^{+0.066}$ & $0.425_{-0.068}^{+0.073}$ & $0.468_{-0.057}^{+0.065}$ & $0.439_{-0.066}^{+0.069}$ \\
\hline$a(\mathrm{AU})$ & $0.04639_{-0.00052}^{+0.00052}$ & $0.04645_{-0.00055}^{+0.00053}$ & $0.04638_{-0.00052}^{+0.00052}$ & $0.04641_{-0.00054}^{+0.00054}$ \\
\hline$T_{\text {eq }}(\mathrm{K})$ & $1623_{-38}^{+39}$ & $1632_{-43}^{+47}$ & $1623_{-37}^{+38}$ & $1624_{-41}^{+44}$ \\
\hline$\Theta^{\mathrm{e}}$ & $0.0761_{-0.0031}^{+0.0032}$ & $0.0737_{-0.0039}^{+0.0038}$ & $0.0761_{-0.0030}^{+0.0031}$ & $0.0745_{-0.0037}^{+0.0035}$ \\
\hline$F_{\text {per }}\left(10^{9} \mathrm{erg} \mathrm{s}^{-1} \mathrm{~cm}^{-2}\right)^{\mathrm{f}}$ & $1.73_{-0.12}^{+0.17}$ & $1.77_{-0.16}^{+0.21}$ & $1.73_{-0.12}^{+0.17}$ & $1.74_{-0.14}^{+0.20}$ \\
\hline$F_{\text {ap }}\left(10^{9} \mathrm{erg} \mathrm{s}^{-1} \mathrm{~cm}^{-2}\right)^{\mathrm{f}}$ & $1.43_{-0.17}^{+0.14}$ & $1.46_{-0.18}^{+0.18}$ & $1.43_{-0.17}^{+0.14}$ & $1.43_{-0.18}^{+0.16}$ \\
\hline$\langle F\rangle\left(10^{9} \mathrm{erg} \mathrm{s}^{-1} \mathrm{~cm}^{-2}\right)^{\mathrm{f}}$ & $1.57_{-0.14}^{+0.16}$ & $1.60_{-0.16}^{+0.19}$ & $1.57_{-0.14}^{+0.15}$ & $1.57_{-0.15}^{+0.18}$ \\
\hline
\end{tabular}

Notes.

${ }^{a} T_{c}$ : Reference epoch of mid transit that minimizes the correlation with the orbital period. It corresponds to $N_{\mathrm{tr}}=-8 . T_{x, y}$ : transit duration between contact points $x$ and $y .(x, y)=(1.5,3.5)$ correspond to the sky-projected center of the planet overlapping the stellar limb.

b Values for a quadratic law and fixed coefficients, adopted from the tabulations by Claret (2004) according to the spectroscopic (SME) parameters listed in Table 4.

${ }^{\mathrm{c}} k \& h$ : Lagrangian orbital parameters derived from the global modeling, and primarily determined by the RV data. $\Psi$ : reciprocal of the scaling factor by which the true stellar density is modified from that found assuming a circular orbit (Kipping 2010).

${ }^{\mathrm{d}}$ Correlation coefficient between the planetary mass $M_{p}$ and radius $R_{p}$.

e The Safronov number is given by $\Theta=\frac{1}{2}\left(V_{\mathrm{esc}} / V_{\mathrm{orb}}\right)^{2}=\left(a / R_{p}\right)\left(M_{p} / M_{\star}\right)$ (see Hansen $\&$ Barman 2007).

${ }^{\mathrm{f}}$ Incoming flux per unit surface area, averaged over the orbit. 
Table 7

Comparison of Four Different Models for the RVs of HAT-P-24b, Described in Section 6.1.3

\begin{tabular}{lccccc}
\hline \hline \multicolumn{1}{c}{ Model } & $d$ & $\chi_{\text {reduced }}^{2}$ & BIC & $\begin{array}{c}\dot{\gamma} \\
\left(\mathrm{m} \mathrm{s}^{-1} \mathrm{day}^{-1}\right)\end{array}$ & $e$ \\
\hline Circular & 2 & 3.72 & 65.3 & $0^{\mathrm{a}}$ & $0^{\mathrm{a}}$ \\
Circular + Drift & 3 & 3.35 & 59.0 & $-0.053_{-0.026}^{+0.026}$ & $0^{\mathrm{a}}$ \\
Eccentric & 4 & 2.83 & 51.2 & $0^{\mathrm{a}}$ & $0.052_{-0.017}^{+0.022}$ \\
Eccentric + Drift & 5 & 2.72 & 49.9 & $-0.040_{-0.028}^{+0.028}$ & $0.048_{-0.017}^{+0.022}$ \\
\hline
\end{tabular}

Note. ${ }^{a}$ Parameter is fixed.

A resolution would be to obtain a secondary eclipse measurement for the system, for which the mid-eclipse time would be dependent upon $e \cos \omega$. The $k$ component dominates the eccentricity budget and thus its determination would strongly constrain the eccentricity of this system.

\subsection{Circularization Timescales}

Tidal dissipation causes planetary orbits to circularize over time. The maximum eccentricity a planet could initially have is $e \sim 1$. After $N=1$ circularization timescales, denoted $\tau_{\text {circ }}$, the planet's eccentricity will reduce by one e-fold, i.e., a factor of 2.72. For the planet to now have an eccentricity of $e$, the number of circularization timescales which have transpired must be $\leqslant-\log (e)$ and therefore $T_{\text {Age }} \leqslant-\log (e) \tau_{\text {circ }}$. This therefore constrains the circularization timescale to be:

$$
\tau_{\text {circ }} \geqslant \frac{T_{\text {Age }}}{-\log (e)}
$$

Using the method of Adams \& Laughlin (2006), the circularization timescale may be expressed as a function of the planet's tidal dissipative constant, $Q_{P}$, for a low eccentricity system.

$$
\tau_{\text {circ }}=Q_{P} \frac{4}{63} \frac{P}{2 \pi} \frac{M_{P}}{M_{*}}\left(\frac{a}{R_{*}} \frac{1}{p}\right)^{5}\left(1-e^{2}\right)^{13 / 2}
$$

Taking $e \simeq 0$ in the above expression together with the posterior distributions of the various parameters given above allows us to constrain $Q_{P}$ to be $Q_{P} \geqslant\left(6.1_{-2.0}^{+3.0}\right) \times 10^{6}$. Note that the value above becomes even larger if we include the $\left(1-e^{2}\right)^{13 / 2}$ term from the Adams \& Laughlin (2006) equation, but this requires some assumption of the history of the system. Given that Jupiter has $Q_{P} \sim 30,000$ (Lainey et al. 2009), this limit raises some questions about why HAT-P-24b has such a large value, somewhat similar to the situation for GJ $436 \mathrm{~b}$ (Deming et al. 2007). In none of our $10^{5}$ realizations do we have a $Q_{P}$ value below 150,000 and therefore in the absence of any eccentricity pumping, a large $Q_{P}$ value is a possible origin for the non-zero eccentricity. This is consistent with the observation of large $Q_{P}$ values in many other known TEPs (Matsumura et al. 2008).

We note that for planets with initial eccentricities $\geqslant 0.2$, the above approximate expressions will be invalid and a full backwards integration of the planet's orbital evolution will be necessary, as pointed out by Leconte et al. (2010). Such a detailed analysis remains outside of the scope of this paper, but our calculations do flag this system as possibly retaining an anomalously large eccentricity requiring further investigation.

\section{LINEAR RV DRIFT}

The unfolded residuals of an eccentric fit seem to hint at a negative linear drift in the RVs. We re-executed the global fit of the data including a RV gradient term $\dot{\gamma}$. We choose to use the $\left\{p^{2}, b, T_{1.5,3.5}\right\}$ parameter set with fitted limb darkening again. The fits obtain $\dot{\gamma}=-0.040_{-0.028}^{+0.028} \mathrm{~m} / \mathrm{s} /$ day with a slightly decreased eccentricity of $e=0.048_{-0.017}^{+0.022}$. By the Lucy \& Sweeney (1971) test, the eccentricity is now significant at the 2.3- $\sigma$ confidence level. We also note that the $\dot{\gamma}$ parameter appears to have converged in the MCMC trials with the GelmanRubins statistic (Gelman \& Rubin 1992) satisfying the criteria of being $<1$ (value was 0.59 ), indicative of good mixing.

\subsection{Statistical Significance}

\subsubsection{F-test}

There are numerous tests which one can employ to evaluate the significance of the gradient. The first one we tried was to compute the F-test between the eccentric orbit and the eccentric orbit + linear drift model. Penalizing for one extra degree of freedom, the F-test find the drift model is accepted with $76.4 \%$ confidence.

\subsubsection{Odds Ratio}

The second test we tried was to extract the posterior distribution of the gradient from the MCMC runs. If the gradient was equal to zero, we would expect $50 \%$ of the MCMC runs to give a positive value and $50 \%$ to give a negative value. In the eccentric + drift model, $f_{\text {neg }}=92.7 \%$ of the MCMC runs gave a negative $\dot{\gamma}$. The odds ratio of the negative valued model over the 50:50 model is:

$$
\mathrm{O}_{\text {drift } / \text { static }}=\frac{0.5}{1-f_{\text {neg }}}
$$

For only two possible models (i.e., a drift or static), the probability of the drift model being the correct one is $\mathrm{P}(\mathrm{drift})=$ $1-\left[1 /\left(1+\mathrm{O}_{\text {drift } / \text { static }}\right)\right]=87.2 \%$. Therefore, both tests so far indicate a $\sim 20 \%$ false alarm probability for the drift model.

\subsubsection{Bayesian Information Criterion}

The final test we performed was to re-fit all of the data using four possible models, using the $\left\{p^{2}, b, T_{1.5,3.5}\right\}$ and fitted-limbdarkening method, each with a different number of degrees of freedom, $d$ :

1. Circular orbit $(d=2)$.

2. Circular orbit + linear drift $(d=3)$.

3. Eccentric orbit $(d=4)$.

4. Eccentric orbit + linear drift $(d=5)$.

In each case, we compute the Bayesian Information Criterion (BIC; Schwarz 1978; Liddle 2007), given by BIC $=\chi^{2}+d \log n$ where $n$ is the number of RV data points. BIC severely penalizes models for having more parameters and offers a statistically valid tool for model selection. We also compute the reduced $\chi^{2}$, given by $\chi_{\text {reduced }}^{2}=\chi^{2} /(n-d)$ (see Table 7 for results). As an example, the circular orbit has only 2 degrees of freedom in $\gamma$ and $K$. One might argue that $P$ and $t_{C}$ are also degrees of freedom but in a global fit, which includes the HAT and FLWO time series, these two parameters are overwhelmingly driven by the photometry and not the RV and thus the RV actually has negligible freedom in these parameters.

The BIC model selection test indicates that the eccentric orbit + linear drift model is the accepted model description of the current RVs when globally fitted with the current photometry for this system. We note that (1) the eccentric models are consistently preferred over the circular orbit models (2) the eccentricity is affected by a negligible degree by including the drift. 
6.1.4. Conclusion

The linear drift model is the preferred model using the BIC. Other tests indicate the model is accepted with a false alarm probability of $20 \%$. This is not sufficient to claim the trend is real and thus we encourage observers to obtain more observations to confirm or reject the existence of this trend.

\subsection{Properties of Putative HAT-P-24c}

We proceed here to constrain the properties of HAT-P-24c under the assumption the trend is real. Whilst this may not turn out to be true, it is useful to consider what the properties of the outer planet would be should the trend be later confirmed.

The period of the outer planet would have to be much greater than the timescale of the observations, or a sinusoidal pattern would have emerged and so $P_{c} \gg 265$ days, most likely of $\mathcal{O} \sim 1000$ days which constrains $a_{c} \gtrsim 2$ AU by Kepler's third law. We note that the habitable zone pushes out as $a_{\text {hab }}=\sqrt{L_{*} / L_{\odot}}$ AU and occurs at $\sim 1.6$ AU for HAT-P-24 and so the outer planet would likely be "cold." Using Equation (1) from Winn et al. (2009), the gradient corresponds to an outer planet satisfying

$$
\frac{M_{c} \sin i_{c}}{a_{c}^{2}}=\frac{|\dot{\gamma}|}{G}=0.082_{-0.056}^{+0.051} M_{J} \mathrm{AU}^{-2} .
$$

Based upon the $a_{c}$ constraint, this therefore implies $M_{c} \sin i_{c} \gtrsim$ $0.3 M_{J}$. Aside from the RVs, there are observational consequences for HAT-P-24b due to the outer planet. The system will behave as an inner-outer binary and thus the outer planet will induce a light-time travel effect, potentially detectable with transit timing variations (TTV) of HAT-P-24b. The inner binary will orbit the barycentre with semi-major axis $\left(a_{c} M_{c} \sin i_{c}\right) /\left(M_{*}+M_{b}\right)$. Therefore, the peak-to-peak light-time effect, for an outer planet of negligible eccentricity, will be

$$
\operatorname{TTV}_{\text {light }}(\text { peak-to-peak }) \simeq \frac{|\dot{\gamma}|}{c} \frac{P_{c}^{2}}{2 \pi^{2}} .
$$

Adding in the best-fit value of $\dot{\gamma}$ gives TTV $_{\text {light }}$ (peak-to-peak $)=0.078\left(P_{c} / \text { years }\right)^{2} \mathrm{~s}$. Therefore, we require $P_{c}>3.6$ years for a $>1 \mathrm{~s} \mathrm{TTV}$ and would need $P_{c}>36$ years for a $>100 \mathrm{~s}$ TTV. We also evaluated the TTV effect due to a distant, perturbing planet, as described in case IV of Agol et al. (2005). We find that a 1000 day period $0.3 M_{J}$ would generate an r.m.s. TTV of $0.008 \mathrm{~s}$ for $e_{c}=0.1,0.06 \mathrm{~s}$ for $e_{c}=0.5$ and $0.8 \mathrm{~s}$ for $e_{c}=0.9$. The challenge of measuring TTVs to this precision over such long timescales is a daunting one and unlikely to reap any reward with current instrumentation.

\section{TRANSIT TIMES}

Whilst the photometric quality of the FLWO data is sufficient for full free fitting, three of the four FLWO light curves are only partial transits and so the errors on the duration and therefore mid-transit time diverge for unconstrained fitting parameters.

A solution to this is to work under the assumption that the duration and depth of the transit do not change from transitto-transit. As a result of the partial transits, a transit duration variation (TDV) analysis is therefore not possible but TTV can be obtained provided it is understood that they are derived as inherently model dependent values, where the model is that of constant duration and depth.

We therefore extract the parameters $p^{2}, b, T, e \sin \omega, e \cos \omega$, $P$, and the linear limb-darkening coefficient $u_{1}$ from the posterior
Table 8

Fitted Mid-transit Times

\begin{tabular}{rcc}
\hline \hline Epoch & $\begin{array}{c}t_{C} /\left(\mathrm{BJD}_{\mathrm{UTC}}-2,450,000\right) \\
(\text { days })\end{array}$ & $\begin{array}{c}\mathrm{O}-\mathrm{C} \\
(\mathrm{s})\end{array}$ \\
\hline-11 & $5206.91085_{-0.00062}^{+0.00062}$ & $-6.9_{-53.6}^{+53.6}$ \\
-9 & $5213.62078_{-0.000065}^{+0.00065}$ & $-55.6_{-56.2}^{+56.2}$ \\
-3 & $5233.75317_{-0.00082}^{+0.00080}$ & $23.1_{-70.8}^{+69.1}$ \\
0 & $5243.81878_{-0.00030}^{+0.00030}$ & $11.9_{-26.0}^{+26.0}$ \\
\hline
\end{tabular}

distribution of the global fit. We select the $\{b, T\}$ parameter set with fitted limb darkening as our favorite solution for this purpose. The free parameters of the individual transit fits are OOT and $t_{C}$. We stress that the parameters assumed to be constant from transit to transit are still allowed to float around their median value with standard deviation given by their derived uncertainties. This ensures that the errors are correctly propagated into the mid-times. The final times are given in Table 8.

Using the linear ephemeris derived from the global fit, including all HAT data, we find that the FLWO transits show no excess variance yielding $\chi^{2}=1.3$ for 2 degrees of freedom. The rms of the four O-C values is $34.7 \mathrm{~s}$. With only four transit times, it is not possible to conduct a meaningful TTV analysis. However, these transits may be used a benchmark for future TTV searches on this system.

\section{FOLLOW-UP POSSIBILITIES}

\subsection{Secondary Eclipse}

The Keck RVs indicate that $e \cos \omega$ is significantly non-zero and thus suggests the secondary eclipse of HAT-P-24b would occur with a timing offset from that a circular orbit. Neglecting terms of order $\cot ^{2} i$, Sterne (1940) shows that the timing offset is given by

$$
\Delta t=\frac{P}{\pi}\left(\frac{e \cos \omega \sqrt{1-e^{2}}}{\left(1-(e \sin \omega)^{2}\right)}+\arctan \left(\frac{e \cos \omega}{\sqrt{1-e^{2}}}\right)\right) .
$$

Using the posterior distribution of parameters from the $\left\{p^{2}, b, T_{1.5,3.5}\right\}$ fitted-limb-darkening MCMC run, we estimate that the secondary eclipse should occur $(1.9 \pm 0.7) \mathrm{hr}$ earlier than that expected for a purely circular orbit. The detection of the eclipse would therefore strongly constrain $e \cos \omega$ which would allow for a revised global fit to the data. Constraining $e \cos \omega$ in this way would allow us to re-compute the statistical significance of the RV linear drift model over the static model.

For Spitzer's $3.6 \mu \mathrm{m}$ and $4.5 \mu \mathrm{m}$ channels, assuming uniform redistribution of energy around the planet and zero albedo, we estimate a depth of $\sim 0.075 \%$ and $\sim 0.10 \%$. At $K_{s}=10.5 \mathrm{mag}$, the brightness is comparable to that of TrES-4 ( $\left.K_{s}=10.3 \mathrm{mag}\right)$ which has been observed with Spitzer and eclipses measured to precisions of $0.011 \%$ and $0.016 \%$ for the two channels respectively (Knutson et al. 2009). What HAT-P-24b lacks in a slighter fainter host star it makes up for with a slightly longer duration than TrES-4b ( 12, 000 s for TrES-4b, see Mandushev et al. 2007, and 13, $150 \mathrm{~s}$ for HAT-P-24b). We therefore estimate that the secondary eclipses will be detectable with $\mathrm{S} / \mathrm{N}$ of $\sim 7$ and $\sim 6$ for $3.6 \mu \mathrm{m}$ and $4.5 \mu \mathrm{m}$, respectively.

\subsection{Transmission Spectroscopy}

Molecular constituents in the terminator of the atmosphere can absorb light and cause the transit depth to increase. The 
spectral variations in the transit depth allow for the detection of molecules within an exoplanet's atmosphere. This depth change can be estimated from first principles by calculating the scale height of the atmosphere and computing the expected change in transit depth using Equation (36) from Winn (2010).

Using the posterior distribution of parameters from the $\{b$, $\left.T_{1.5,3.5}\right\}$ fitted-limb-darkening MCMC run, we estimate that $\Delta \delta \simeq(0.014 \pm 0.001) N_{\mathrm{H}} \%$ where $N_{\mathrm{H}}$ is the number of scale heights of atmosphere absorbed by the molecular species (of order unity), and we have used $\mu_{M}=2$ a.m.u for $H_{2}$. Using the same Spitzer uncertainty estimates from before, we would require $N_{\mathrm{H}} \sim 3$ for even $\mathrm{H}_{2}$ to be detectable. Therefore, HAT$\mathrm{P}-24 \mathrm{~b}$ would likely be a challenging target for transmission spectroscopy.

\subsection{Rossiter-McLaughlin Effect}

Owing to HAT-P-24's relatively rapid stellar rotation, a large Rossiter-McLaughlin effect (Rossiter 1924; McLaughlin 1924) amplitude is expected. Equally, a large signal for spectral line tomography could be detectable (Cameron et al. 2010). Using Equation (40) from Winn (2010), and the posterior distribution of parameters from the $\left\{b, T_{1.5,3.5}\right\}$ fitted-limbdarkening MCMC run, we estimate $\Delta V_{\mathrm{RM}}=(95 \pm 5) \mathrm{m}$ $\mathrm{s}^{-1}$. Given the RV measurements are essentially jitter-limited at $7.2 \mathrm{~m} \mathrm{~s}^{-1}$, this indicates that we expect a very large signal-to-noise for the RM effect of HAT-P-24b, reaching $\mathrm{S} / \mathrm{N} \sim 13$.

Another motivation for measuring the planet's RM effect is that HAT-P-24 has an effective temperature of $T_{\text {eff }}=(6373 \pm$ 80 ) $\mathrm{K}$ and thus lies above the $\sim 6250 \mathrm{~K}$ threshold for which most system seem to exhibit significant obliquities (Winn et al. 2010). This therefore indicates that we can not only expect a very large RM effect but also possibly a highly oblique configuration.

Gaudi \& Winn (2007) showed that $v \sin i$ and $\lambda$ become degenerate for low impact parameter transits and so the large RM amplitude predicted for this system should help in solving for the system parameters.

\section{SUMMARY}

We announce the detection of a $0.68 M_{J}$ transiting exoplanet on a 3.36 day orbit around an F8 star (system parameters are found in the last column of Table 6). We find that the planet retains a small eccentricity of $e=0.052_{-0.017}^{+0.022}$ with a $5.8 \%$ false alarm probability, which may suggest either a perturbing planet in the system or low tidal dissipation within the planet of $Q_{P} \gtrsim(6 \pm 3) \times 10^{6}$. Most of the eccentricity originates from the $e \cos \omega$ term and thus we predict that a secondary eclipse observation, which is shown to be feasible, should confirm/ reject the eccentricity hypothesis conclusively.

We have performed a detailed investigation of the effects upon the system parameters by using different fitting sets. In three different parameter sets, we find a consistent solution indicating the result is not sensitive to the priors. The effects of fixingversus fitting-limb-darkening coefficients are also investigated, which leads to slightly increased error bars but a consistent best-fit solution.

Using the Bayesian Information Criterion as a model selection tool, we find the Keck RVs are best described by a model consisting of non-zero orbital eccentricity and a negative linear drift of $(-14.6 \pm 10.2) \mathrm{m} \mathrm{s}^{-1}$ year $^{-1}$ with a false alarm probability of $20 \%$. We consider this trend to be currently not statistically significant, but warranting further investigation.
HAT-P-24 has a relatively rapid stellar rotation of $v \sin i=$ $(10.0 \pm 0.5) \mathrm{km} \mathrm{s}^{-1}$, and we therefore predict HAT-P-24b should exhibit one of the largest known Rossiter-McLaughlin effect amplitudes for an exoplanet $\left(\Delta V_{\mathrm{RM}} \simeq 95 \mathrm{~m} \mathrm{~s}^{-1}\right)$. Further, it has recently been suggested by Winn et al. (2010) that hot stars have companions on preferentially oblique orbits and so HAT-P-24 $\left(T_{\text {eff }}=(6373 \pm 80) \mathrm{K}\right)$ would be an excellent target to further investigate this hypothesis.

HATNet operations have been funded by NASA grants NNG04GN74G, NNX08AF23G and SAO IR\&D grants. DK was supported by STFC and as an SAO Predoctoral Fellow. Work of G.Á.B. and J. Johnson were supported by the Postdoctoral Fellowship of the NSF Astronomy and Astrophysics Program (AST-0702843 and AST-0702821, respectively). GT acknowledges partial support from NASA grant NNX09AF59G. We acknowledge partial support also from the Kepler Mission under NASA Cooperative Agreement NCC2-1390 (D.W.L., PI). G.K. thanks the Hungarian Scientific Research Foundation (OTKA) for support through grant K-81373. This research has made use of Keck telescope time granted through NOAO and NASA. This paper uses observations obtained with facilities of the Las Cumbres Observatory Global Telescope.

\section{REFERENCES}

Adams, F. C., \& Laughlin, G. 2006, ApJ, 649, 1004

Agol, E., Steffen, J., Sari, R., \& Clarkson, W. 2005, MNRAS, 359, 567

Bakos, G. Á., Noyes, R. W., Kovács, G., Stanek, K. Z., Sasselov, D. D., \& Domsa, I. 2004, PASP, 116, 266

Bakos, G. Á., et al. 2007, ApJ, 670, 826

Bakos, G. Á., et al. 2010, ApJ, 710, 1724

Butler, R. P., et al. 1996, PASP, 108, 500

Cameron, A. C., et al. 2010, MNRAS, 407, 507

Carpenter, J. M. 2001, AJ, 121, 2851

Carter, J. A., \& Winn, J. N. 2010, ApJ, 709, 1219

Charbonneau, D., Brown, T. M., Latham, D. W., \& Mayor, M. 2000, ApJ, 529, L45

Claret, A. 2004, A\&A, 428, 1001

Deming, D., Harrington, J., Laughlin, G., Seager, S., Navarro, S. B., Bowman, W. C., \& Horning, K. 2007, ApJ, 667, L199

Droege, T. F., Richmond, M. W., \& Sallman, M. 2006, PASP, 118, 1666

Ford, E. 2006, ApJ, 642, 505

Gaudi, S. B., \& Winn, J. N. 2007, ApJ, 655, 550

Gelman, A., \& Rubin, D. B. 1992, Stat. Sci., 7, 457

Hansen, B. M. S., \& Barman, T. 2007, ApJ, 671, 861

Hartman, J. D., et al. 2009, ApJ, 706, 785

Johnson, J. A., Aller, K. M., Howard, A. W., \& Crepp, J. R. 2010, PASP, 122, 905

Kipping, D. M. 2010, MNRAS, 407, 301

Knutson, H. A., Charbonneau, D., Burrows, A., O’Donovan, F. T., \& Mandushev, G. 2009, ApJ, 691, 866

Knutson, H. A., et al. 2007, Nature, 447, 183

Kovács, G., Bakos, G. Á., \& Noyes, R. W. 2005, MNRAS, 356, 557

Kovács, G., Zucker, S., \& Mazeh, T. 2002, A\&A, 391, 369

Kovács, G., et al. 2010, ApJ, 724, 866

Lainey, V., Arlot, J. E., Karatekin, O., \& Hoolst, T. V. 2009, Nature, 459, 957

Latham, D. W. 1992, in ASP Conf. Ser. 32, IAU Colloq. 135, Complementary Approaches to Double and Multiple Star Research, ed. H. A. McAlister \& W. I. Hartkopf (San Francisco, CA: ASP), 110

Leconte, J., Chabrier, G., Baraffe, I., \& Levrard, B. 2010, A\&A, 516, L64

Liddle, A. R. 2007, MNRAS, 377, L74

Mandushev, G., et al. 2007, ApJ, 686, L195

Matsumura, S., Takeda, G., \& Rasio, F. A. 2008, ApJ, 686, L29

Pál, A. 2009a, MNRAS, 396, 1737

Pál, A. 2009b, PhD thesis, Department of Astronomy, Eötvös Loránd University (arXiv:0906.3486)

Pál, A., \& Bakos, G. Á. 2006, PASP, 118, 1474

Press, W. H., Teukolsky, S. A., Vetterling, W. T., \& Flannery, B. P. 1992, Numerical Recipes in C: the Art of Scientific Computing (2nd ed.; Cambridge: Cambridge Univ. Press) 
Lucy, L. B., \& Sweeney, M. A. 1971, AJ, 76, 544

Mandel, K., \& Agol, E. 2002, ApJ, 580, L171

Marcy, G. W., \& Butler, R. P. 1992, PASP, 104, 270

McLaughlin, D. B. 1924, ApJ, 60, 22

Queloz, D., et al. 2001, A\&A, 379, 279

Rossiter, R. A. 1924, ApJ, 60, 15

Schwarz, G. 1978, Ann. Stat., 6, 461

Seager, S., \& Hui, L. 2002, ApJ, 575, 1004

Seager, S., \& Mallén-Ornelas, G. 2003, ApJ, 585, 1038

Shkolnik, E., Bohlender, D. A., Walker, G. A. H., \& Collier Cameron, A. 2008, ApJ, 676, 628

Skrutskie, M. F., et al. 2006, AJ, 131, 1163

Sterne, T. E. 1940, PNAS, 26, 36
Tingley, B., \& Sackett, P. D. 2005, ApJ, 627, 1011

Torres, G., Boden, A. F., Latham, D. W., Pan, M., \& Stefanik, R. P. 2002, AJ, 124,1716

Torres, G., et al. 2007, ApJ, 666, 121

Valenti, J. A., \& Fischer, D. A. 2005, ApJS, 159, 141

Valenti, J. A., \& Piskunov, N. 1996, A\&AS, 118, 595

Vaughan, A. H., Preston, G. W., \& Wilson, O. C. 1978, PASP, 90, 267

Vogt, S. S., et al. 1994, Proc. SPIE, 2198, 362

Winn, J. N. 2010, arXiv:1001.2010

Winn, J. N., Fabrycky, D., Albrecht, S., \& Johnson, J. A. 2010, ApJ, 718, L145

Winn, J. N., Johnson, J. A., Albrecht, S., Howard, A. W., Marcy, G. W., Crossfield, I. J., \& Holman, M. J. 2009, ApJ, 703, 99

Yi, S. K., et al. 2001, ApJS, 136, 417 